\title{
Strengthening the reporting of observational studies in epidemiology (STROBE) statement: guidelines for reporting observational studies
}

\author{
Poor reporting of research hampers assessment and makes it less useful. An international group \\ of methodologists, researchers, and journal editors sets out guidelines to improve reports of \\ observational studies
}

Many questions in medical research are investigated in observational studies. ${ }^{1}$ Much of the research into the cause of diseases relies on cohort, case-control, or cross sectional studies. Observational studies also have a role in research into the benefits and harms of medical interventions. ${ }^{2}$ Randomised trials cannot answer all important questions about a given intervention. For example, observational studies are more suitable to detect rare or late adverse effects of treatments, and are more likely to provide an indication of what is achieved in daily medical practice. ${ }^{3}$

Research should be reported transparently so that readers can follow what was planned, what was done, what was found, and what conclusions were drawn. The credibility of research depends on a critical assessment by others of the strengths and weaknesses in study design, conduct, and analysis. Transparent reporting is also needed to judge whether and how results can be included in systematic reviews. ${ }^{4}$ However, in published observational research important information is often missing or unclear. An analysis of epidemiological studies published in general medical and specialist journals found that the rationale behind the choice of potential confounding variables was often not reported ${ }^{6}$ Only few reports of case-control studies in psychiatry explained the methods used to identify cases and controls. ${ }^{7}$ In a survey of longitudinal studies in stroke research, 17 of 49 articles (35\%) did not specify the eligibility criteria. ${ }^{8}$ Others have argued that without sufficient clarity of reporting, the benefits of research might be achieved more slowly, ${ }^{9}$ and that there is a need for guidance on reporting observational studies..$^{10} 11$

Recommendations on the reporting of research can improve reporting quality. The consolidated standards of reporting trials (CONSORT) statement was developed in 1996 and revised five years later. ${ }^{12}$ Many medical journals supported this initiative, ${ }^{13}$ which has helped to improve the quality of reports of randomised trials. ${ }^{14}{ }^{15}$ Similar initiatives have followed for other research areas-for example, for the reporting of metaanalyses of randomised trials ${ }^{16}$ or diagnostic studies. ${ }^{17}$ We established a network of methodologists, researchers, and journal editors to develop recommendations for reporting observational research: the strengthening the reporting of observational studies in epidemiology (STROBE) statement.
Editorial by Rothwell and Bhatia Erik von Elm senior research fellow, Matthias Egger professor, Institute of Social and Preventive Medicine, University of Bern, Finkenhubelweg 11, CH-3012 Bern, Switzerland

Douglas G Altman professor Centre for Statistics in Medicine, University of Oxford, Oxford Stuart J Pocock professor, London School of Hygiene and Tropical Medicine, University of London, London

Peter C Gøtzsche director, Nordic Cochrane Centre, Copenhagen, Denmark

Jan P Vandenbroucke professor, Department of Clinical Epidemiology, Leiden University Hospital, Leiden, Netherlands

for the STROBE Initiative Correspondence to: Evon Elm strobe@ispm.unibe.ch

\section{Aims and use of STROBE statement}

The STROBE statement is a checklist of items that should be addressed in articles reporting on the three main study designs of analytical epidemiology: cohort, case-control, and cross sectional studies. The intention is solely to provide guidance on how to report observational research well: these recommendations are not prescriptions for designing or conducting studies. Also, while clarity of reporting is a prerequisite to evaluation, the checklist is not an instrument to evaluate the quality of observational research.

Here we present the STROBE statement and explain how it was developed. In a detailed companion article, the explanation and elaboration article, ${ }^{18-20}$ we justify the inclusion of the different checklist items and give methodological background and published examples of what we consider transparent reporting. We strongly recommend using the STROBE checklist in conjunction with the explanatory article, which is available freely on the websites of the publishing journals. ${ }^{18-20}$

\section{Development of STROBE statement}

We established the STROBE Initiative in 2004, obtained funding for a workshop and set up a website (www.strobe-statement.org). We searched textbooks, bibliographic databases, reference lists, and personal files for relevant material, including previous recommendations, empirical studies of reporting, and articles describing relevant methodological research. Because observational research makes use of many different study designs, we felt that the scope of STROBE had to be clearly defined early on. We decided to focus on the three study designs that are used most widely in analytical observational research: cohort, case-control, and cross sectional studies.

We organised a two day workshop in Bristol in September 2004. Twenty three people attended this meeting, including editorial staff from Annals of Internal Medicine, BMJ, Bulletin of the World Health Organization, International Journal of Epidemiology, JAMA, Preventive Medicine, and the Lancet as well as epidemiologists, methodologists, statisticians, and practitioners from Europe and North America. Written contributions were sought from 10 other people who declared an interest in contributing to STROBE but could not attend.

Three working groups identified items that were 
deemed to be important to include in checklists for each type of study. A provisional list of items prepared in advance (available from our website) was used to facilitate discussions. The three draft checklists were then discussed by all participants and, where possible, items were revised to make them applicable to all three study designs. In a final plenary session, the group decided on the strategy for finalising and disseminating the STROBE statement.

After the workshop we drafted a combined checklist including all three designs and made it available on our website. We invited participants and additional scientists

STROBE statement-checklist of items that should be included in reports of observational studies

\begin{tabular}{|c|c|}
\hline Item No & Recommendation \\
\hline Title and abstract & \\
\hline
\end{tabular}

1 (a) Indicate the study's design with a commonly used term in the title or the abstract

(b) Provide in the abstract an informative and balanced summary of what was done and what was found

\section{Introduction}

Background/rationale 2 Explain the scientific background and rationale for the investigation being reported

Objectives 3 State specific objectives, including any prespecified hypotheses

$\begin{array}{lll}\text { Methods } & \\ \text { Study design } & 4 & \text { Present key elements of study design early in the paper }\end{array}$

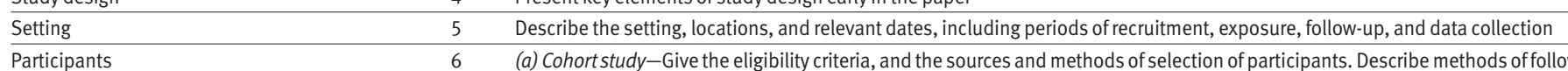

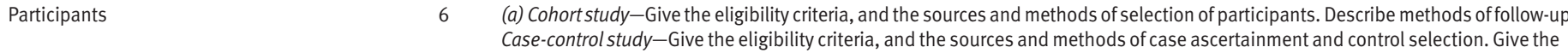
rationale for the choice of cases and controls

Cross sectional study-Give the eligibility criteria, and the sources and methods of selection of participants

(b) Cohortstudy-For matched studies, give matching criteria and number of exposed and unexposed Case-control study-For matched studies, give matching criteria and the number of controls per case

\begin{tabular}{|c|c|c|}
\hline Variables & 7 & Clearly define all outcomes, exposures, predictors, potential confounders, and effect modifiers. Give diagnostic criteria, if applicable \\
\hline Data sources/measurement & $8^{\star}$ & $\begin{array}{l}\text { For each variable of interest, give sources of data and details of methods of assessment (measurement). Describe comparability of } \\
\text { assessment methods if there is more than one group }\end{array}$ \\
\hline Bias & 9 & Describe any efforts to address potential sources of bias \\
\hline Study size & 10 & Explain how the study size was arrived at \\
\hline Quantitative variables & 11 & Explain how quantitative variables were handled in the analyses. If applicable, describe which groupings were chosen and why \\
\hline \multirow[t]{5}{*}{ Statistical methods } & \multirow[t]{5}{*}{12} & (a) Describe all statistical methods, including those used to control for confounding \\
\hline & & (b) Describe any methods used to examine subgroups and interactions \\
\hline & & (c) Explain how missing data were addressed \\
\hline & & $\begin{array}{l}\text { (d) Cohort study-If applicable, explain how loss to follow-up was addressed } \\
\text { Case-control study-If applicable, explain how matching of cases and controls was addressed } \\
\text { Cross sectional study-If applicable, describe analytical methods taking account of sampling strategy }\end{array}$ \\
\hline & & (e) Describe any sensitivity analyses \\
\hline
\end{tabular}

\section{Results}

Participants $\quad 13^{\star} \quad$ (a) Report numbers of individuals at each stage of study-eg numbers potentially eligible, examined for eligibility, confirmed eligible, included in the study, completing follow-up, and analysed

(b) Give reasons for non-participation at each stage

(c) Consider use of a flow diagram

\begin{tabular}{|c|c|c|}
\hline \multirow[t]{3}{*}{ Descriptive data } & \multirow[t]{3}{*}{$14^{*}$} & (a) Give characteristics of study participants (eg demographic, clinical, social) and information on exposures and potential confounders \\
\hline & & (b) Indicate number of participants with missing data for each variable of interest \\
\hline & & (c) Cohortstudy-Summarise follow-up time (eg average and total amount) \\
\hline \multirow[t]{3}{*}{ Outcome data } & \multirow[t]{3}{*}{$15^{\star}$} & Cohortstudy-Report numbers of outcome events or summary measures over time \\
\hline & & Case-control study-Report numbers in each exposure category, or summary measures of exposure \\
\hline & & Cross sectional study-Report numbers of outcome events or summary measures \\
\hline \multirow[t]{3}{*}{ Main results } & \multirow[t]{3}{*}{16} & $\begin{array}{l}\text { (a) Report the numbers of individuals at each stage of the study-eg numbers potentially eligible, examined for eligibility, confirmed } \\
\text { eligible, included in the study, completing follow-up, and analysed }\end{array}$ \\
\hline & & (b) Give reasons for non-participation at each stage \\
\hline & & (c) Consider use of a flow diagram \\
\hline Other analyses & 17 & Report other analyses done-eg analyses of subgroups and interactions, and sensitivity analyses \\
\hline \multicolumn{3}{|l|}{ Discussion } \\
\hline Key results & 18 & Summarise key results with reference to study objectives \\
\hline Limitations & 19 & $\begin{array}{l}\text { Discuss limitations of the study, taking into account sources of potential bias or imprecision. Discuss both direction and magnitude } \\
\text { of any potential bias }\end{array}$ \\
\hline Interpretation & 20 & $\begin{array}{l}\text { Give a cautious overall interpretation of results considering objectives, limitations, multiplicity of analyses, results from similar } \\
\text { studies, and other relevant evidence }\end{array}$ \\
\hline Generalisability & 21 & Discuss the generalisability (external validity) of the study results \\
\hline \multicolumn{3}{|l|}{ Other information } \\
\hline Funding & 22 & $\begin{array}{l}\text { Give the source of funding and the role of the funders for the present study and, if applicable, for the original study on which the } \\
\text { present article is based }\end{array}$ \\
\hline
\end{tabular}

*Give information separately for cases and controls in case-control studies and, if applicable, for exposed and unexposed groups in cohort and cross sectional studies.

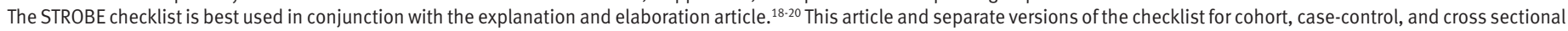
studies are available at www.strobe-statement.org. 
and editors to comment on this draft checklist. We subsequently published three revisions.

\section{STROBE components}

The STROBE statement is a checklist of 22 items that we consider essential for good reporting of observational studies (table). These items relate to the article's title and abstract (item 1), the introduction (items 2 and 3 ), methods (items 4-12), results (items 13-17), discussion sections (items 18-21), and other information (item 22 on funding). Eighteen items are common to all three designs, while four (items 6, 12, 14, and 15) are design specific, with different versions for all or part of the item. For some items (indicated by asterisks), information should be given separately for cases and controls in case-control studies, or exposed and unexposed groups in cohort and cross sectional studies. Although the table is a single checklist, the STROBE website provides separate checklists for each of the three study designs.

\section{Implications and limitations}

The STROBE statement was developed to assist authors when writing up analytical observational studies, to support editors and reviewers when considering such articles for publication, and to help readers when critically appraising published articles.

Observational studies serve a wide range of purposes, on a continuum from the discovery of new findings to the confirmation or refutation of previous findings. ${ }^{18-20}$ Some studies are essentially exploratory and raise interesting hypotheses. Others pursue clearly defined hypotheses in available data. In yet another type of studies, the collection of new data is planned carefully on the basis of an existing hypothesis. We believe the present checklist can be useful for all these studies, since the readers always need to know what was planned (and what was not), what was done, what was found, and what the results mean.

We acknowledge that STROBE is currently limited to three main observational study designs. We would welcome extensions that adapt the checklist to other designs-for example, case crossover studies or ecological studies-and also to specific topics. Four extensions are now available for the CONSORT statement. ${ }^{21-24}$ A first extension to STROBE is under way for gene-disease association studies: the STROBE Extension to Genetic Association studies (STREGA) initiative.$^{25}$ We ask those who aim to develop extensions of the STROBE statement to contact the coordinating group first to avoid duplication of effort.

The STROBE statement should not be interpreted as an attempt to prescribe the reporting of observational research in a rigid format. The checklist items should be addressed in sufficient detail and with clarity somewhere in an article, but the order and format for presenting information depends on author preferences, journal style, and the traditions of the research field. For instance, we discuss the reporting of results under a number of separate items, while recognising that authors might address several items within a single section of text or in a table. Also, item
22 , on the source of funding and the role of funders, could be addressed in an appendix or in the methods section of the article. We do not aim at standardising reporting. Authors of randomised clinical trials were asked by an editor of a specialist medical journal to "CONSORT" their manuscripts on submission. ${ }^{26} \mathrm{We}$ believe that manuscripts should not be "STROBEd," in the sense of regulating style or terminology. We encourage authors to use narrative elements, including the description of illustrative cases, to complement the essential information about their study, and to make their articles an interesting read. ${ }^{27}$

We emphasise that the STROBE statement was not developed as a tool for assessing the quality of published observational research. Such instruments have been developed by other groups and were the subject of a recent systematic review. ${ }^{28}$ In the explanatory article we used several examples of good reporting from studies whose results were not confirmed in further research; the important feature was the good reporting, not the quality of the research. However, if authors and journals adopt the STROBE statement, issues such as confounding, bias, and generalisability could become more transparent, which might help temper the overenthusiastic reporting of new findings in the scientific community and popular media, ${ }^{29}$ and improve the methods of studies in the long term. Better reporting may also help to have more informed decisions about when new studies are needed, and what they should address.

We did not undertake a comprehensive systematic review for each of the checklist items and subitems, or do our own research to fill gaps in the evidence base. Furthermore, although no one was excluded from the process, the composition of the group of contributors was influenced by existing networks and was not representative in terms of geography and probably was not representative in terms of research interests and disciplines. We stress that STROBE and other recommendations on the reporting of research should be seen as evolving documents that require continual assessment, refinement, and, if necessary, change. We welcome suggestions for the further dissemination of STROBE-for example, by republishing this article in specialist journals and in journals published in other languages. Groups or individuals who intend to translate the checklist to other languages should consult the coordinating group beforehand. We will revise the checklist in the future, taking into account comments, criticism, new evidence, and experience from its use. We invite readers to submit their comments through the STROBE website.

To encourage dissemination of the STROBE statement, this article is free on bmj.com and is also published and freely available in Annals of Internal Medicine, Bulletin of the World Health Organization, Epidemiology, the Lancet, PLOS Medicine, and Preventive Medicine. For details on further use, see STROBE website (www.strobe-statement.org).

Funding: The workshop was funded by the European Science Foundation. The UK Medical Research Council's Health Services Research Collaboration and the NHS research and development methodology programme provided additional funding.

Competing interests: None declared

Contributors and all references are on bmj.com 